\title{
An Electronically Tunable Resonator for Noise Control
}

\author{
Clark J. Radcliffe \\ Mechanical Engineering \\ Michigan State University \\ Charles Birdsong \\ Dactron, Inc.
}

Copyright $@ 2000$ Society of Automotive Engineers, Inc.

\begin{abstract}
Many engineering systems create unwanted noise that can be reduced by the careful application of engineering noise controls. When this noise travels down tubes and pipes, a tuned resonator can be used to muffle noise escaping from the tube. The classical examples are automobile exhaust and ventilation system noise. In these cases where a narrow frequency band of noise exists, a traditional engineering control consists of adding a tuned Helmholtz resonator to reduce unwanted tonal noise by reflecting it back to the source (Temkin, 1981). As long as the frequency of the unwanted noise falls within the tuned resonator frequency range, the device is effective. However, if the frequency of the unwanted sound changes to a frequency that does not match the tuned resonator frequency, the device is no longer effective. Conventional resonators have fixed tuning and cannot effectively muffle tonal noise with time-varying frequency.
\end{abstract}

The electronically tunable Semi-active Helmholtz Resonator (SHR) is a novel noise control device that includes a combination of conventional acoustic components combined with a hybrid control system. This device can be fully designed externally and attached to a primary acoustic system, such as a duct or pipe to reduce the transmission of tonal noise. It is adaptively tuned on-line to track a disturbance signal with slow time varying frequency. The paper presents a physical analytical model of the SHR, a power flow model that answers the question "Where does the power go?" and finally demonstrates the effectiveness of the device with experimental test results.

\section{INTRODUCTION}

Helmholtz resonators are commonly used to reduce sound from transmitting through acoustic systems such as industrial processes, wind tunnels (Heidelberg, and Gordon, 1989), vehicle exhaust (Hsomi, et. al., 1993), industrial ducting systems, and more. They function by reflecting sound in a narrow, tuned frequency band back to the source, where the tuned frequency is a function of the dimensions of the resonator cavity (Pierce, 1981). If the frequency of the unwanted noise changes from the tuned value, the noise reduction is diminished. Past efforts at active tuning of Helmholtz resonators resulted in limited tuning capability and significant mechanical complexity (Bedout, 1997). This work considers a system that modifies the acoustic response of a Helmholtz resonator continuously through simple adaptive control, that allows optimum performance over a range of operating conditions. The system consists of a static Helmholtz resonator designed to enforce a nominal resonance and a hybrid feedback control system that provides a variable acoustic impedance. The system is termed "semi-active" because the objective of the hybrid control is retuning of resonator resonances - not control of the acoutic noise. The combination of the nominal impedance of the resonator and the differential impedance of the controller results in a semi-active controlled, variable frequency Helmholtz resonator.

A closed-loop adaptive control strategy is summarized in this article for implementing the Semi-Active Helmholtz Resonator (SHR) (Birdsong, 1999). This device is tuned with an automated algorithm to modify the resonant frequency and amplitude of the resonator to reflect sound back to the source at a single command frequency, selected to match the frequency of the unwanted noise. The objective is a self-tuning acoustic resonator that will track a pure tone noise source with a time varying frequency. The time varying signals tested were of a pure tone with a frequency that changed at a rate on the order of $1 \mathrm{~Hz} / \mathrm{sec}$ which is typical of many tonal noise control applications for sound propagating down a tube or duct.

A theoretical model of the system with an ideal actuator is presented and an analytical controller design is presented. Actuator internal dynamics degrade the performance of the analytical controller design. This motivates the use of a model based empirical controller design based on qualitative information learned from the 
model. A gain scheduled adaptive controller is developed using this design technique. Numerical simulations and experimental results are given to demonstrate the effectiveness of the system.

\section{ANALYTICAL SEMI-ACTIVE HELMOLTZ RESONATOR MODEL}

The Helmholtz resonator is a classic acoustic device which consists of a rigid-wall acoustic cavity with at least one short and narrow orifice, or "neck" through which the fluid filling it communicates with the external medium (Figure 1). Temkin, (1936) developed a model to obtain the impedance of an acoustic resonator. He studied the action of a monochromatic wave on the device, under the assumption the lateral dimensions of the cavity were small compared with the wavelength of the incident wave. The cavity creates an acoustic compliance that can be computed from the physical dimensions of the resonator as

$$
C_{a}=\frac{V}{\rho_{b} c_{b}^{2}}\left(\mathrm{~m}^{5} \mathrm{~N}\right)
$$

where $V$ is the cavity volume, $\rho_{o}$ is the density of the medium, and $c_{o}$ is the speed of sound in the medium.

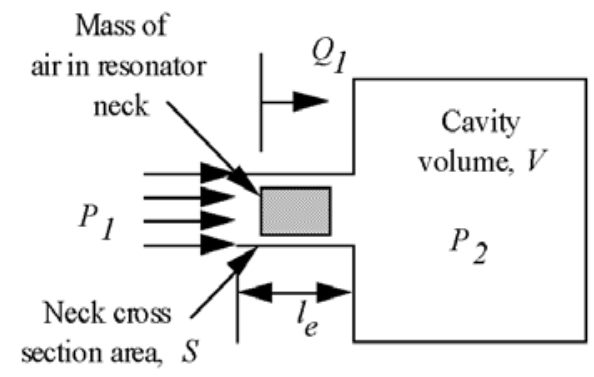

Figure 1: Ideal Helmholtz Resonator (Birdsong, 1999)

The mass of air in the neck will oscillate in response to the wave as a solid body with effective inertia

$$
l_{a}=\frac{\rho_{Q} l_{o}}{S}\left(\mathrm{Ns}^{2} \mathrm{~m}^{5}\right)
$$

where $l_{e}$ and $S$ are the effective length and cross sectional area of the neck. When dissipation is small, resonance occurs at a frequency

$$
\left(n_{n}=\sqrt{1 / C_{n} l_{n}}(\text { rads } \mathrm{s})\right.
$$

Summing the forces on the inertia produces a second order differential equation relating the pressure, $P_{I}$ $\left(\mathrm{N} / \mathrm{m}^{2}\right)$, at the entrance of the neck to the volumetric flow rate, or "volume velocity," $\left(\mathrm{m}^{3} / \mathrm{s}\right)$. Temkin's model is extended here by converting the differential equation into the transfer function model

$$
\frac{O_{1}}{P_{1}}=\frac{1}{I_{s}}\left[\frac{s}{s^{2}+\frac{R_{s}}{I_{a}} s+\frac{1}{C_{a} I a}}\right]
$$

where $R_{a}$ is the resistance due to radiation losses and viscous damping of the medium.

The ideal Helmholtz resonator model can be modified by adding a boundary condition to the cavity interior surface, relating the surface volume velocity, $Q_{2}$, to the pressure acting on the surface, $P_{2}$ (Radcliffe and Gogate, 1994) as

$$
\frac{P_{7}}{Q_{2}}=Z_{2}(s)
$$

where $Z_{2}$ is an arbitrary acoustic impedance. Figure 2 shows a Helmholtz resonator with this boundary condition added.

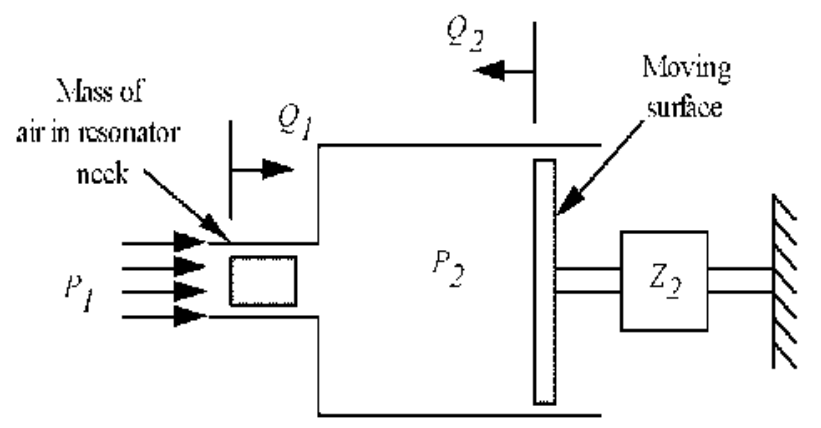

Figure 2. Semi-Active Helmholtz Resonator (SHR) with impedance boundary condition, $Z_{2}$ (Birdsong, 1999)

The SHR transfer function resulting from this modification can then be found (Birdsong, 1999) is

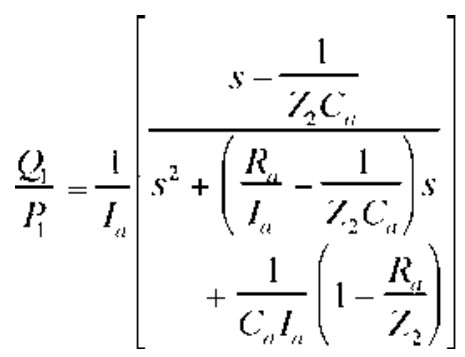

The SHR uses a feedback control system to modify the resonant dynamic response of the system by specifying $Z_{2}$. In particular, a specific value of $Z_{2}$ can be found that produces a resonance in the $Q_{I} / P_{I}$ transfer function at an arbitrary frequency with arbitrary peak magnitude. Solving (6) for $Z_{2}$ and replacing $Q_{2} / P_{2}$ with a constant, $x_{o}$, which specifies the height of the resonant peak, and letting $s=j \omega_{C}$ gives 


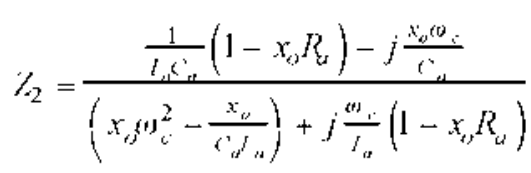

where $\omega_{C}$ is a scalar value that represents the frequency of the desired resonant peak. This impedance can be separated into real and imaginary parts by multiplying the complex conjugate of the denominator of (7) and collecting real and imaginary parts.

Assuming that the disturbance is a narrow frequency band tone at a relatively low frequency, $Z_{2}$ can be implemented with a positive feedback, proportionalintegral (PI) controller. A positive feedback arrangement is used to keep the sign conventions consistent with Figure 2. A PI controller is ideal because it is insensitive to noise, it is generally stable, and it has characteristics that are well understood. The $\mathrm{PI}$ controller is not suitable for higher frequencies because the magnitude of the integral signal falls off with increasing frequency. Under this assumption, (7) need not be implemented for all frequencies. Instead, the controller can implement a single valued complex impedance which corresponds to the disturbance frequency. The gain, $K_{I}$, must be increased with frequency to compensate for the attenuation that occurs with frequency in an integral controller. Note the controller outputs a $Q_{2}$ for an input $P_{2}$, therefore the controller transfer function is the inverse of $Z_{2}$. The control law is given by

$$
G(s)=\frac{Q_{3}}{P_{3}}=K_{p}+\frac{\Lambda_{1}}{s}
$$

where the gains $K_{P}$ and $K_{I}$ are given by

$$
\begin{aligned}
& K_{l}=\operatorname{Rc}\left(\frac{1}{\alpha_{3}}\right) \\
& K_{I}=\omega^{*} \operatorname{Im}\left(\frac{1}{\alpha_{a}}\right)
\end{aligned}
$$

This produces an analytical controller design based on computing the controller gains $K_{P}$ and $K_{I}$ from the relation for (7) for desired values of $\omega_{C}$ and $x_{0}$.

The closed loop transfer function in terms of controller gains $K_{P}$ and $K_{I}$ can be computed by replacing $Z_{2}$ with the inverse of $G_{c}$ in (6) and simplifying.

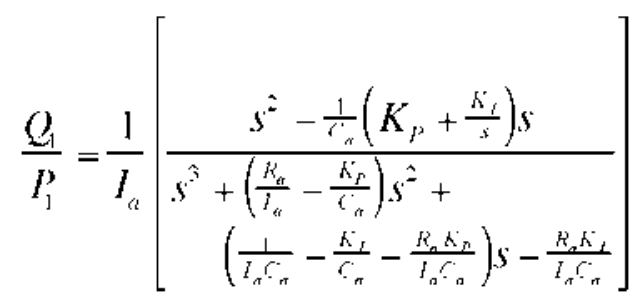

Table 1. Controller Gains for Simulation with Actuator Dynamics (Birdsong, 1999)

\begin{tabular}{|c|c|c|c|}
\hline Graph & $\begin{array}{c}\text { Resonant } \\
\text { Frequency } \\
(\mathrm{Hz})\end{array}$ & $\begin{array}{c}K_{P} \\
\text { Gain }\end{array}$ & $\begin{array}{c}K_{I} \\
\text { Gain }\end{array}$ \\
\hline A & 112 & 0.99 & -100 \\
\hline B & 130 & 0.99 & 0 \\
\hline C & 145 & 0.99 & 100 \\
\hline
\end{tabular}

This form of the transfer function shows how the controller gains effect the system response. Although the SHR system is not a simple second order resonator, $K_{I}$ dominantly changes the effective resonant frequency and $K_{P}$ dominantly affects the apparent acoustic loss. In general, positive $K_{I}$ decreases the effective resonant frequency while a positive $K_{P}$ decreases the system damping. Reducing system damping increases the peak magnitude at resonance increasing SHR acoustic effectiveness at the resonant frequency. The SHR is termed "Semi-active" because the SHR only interacts through the controllable cavity acoustic impedance, $Z_{2}$, to change the Helmholtz resonator natural frequency and damping.

Acoustic actuators always have finite dynamics that affect SHR system performance controller design. The previous analysis assumed that the actuator transfer function was a pure gain. Unfortunately, most commercially available acoustic actuators such as voice coil speakers do not have such ideal response characteristics. Birdsong and Radcliffe (1999) presented an actuator that uses feedback compensation to improve the response of a dual voice coil speaker by compensating for the internal dynamics and the pressure interaction with the acoustic system. While this actuator does not have a transfer function that is a pure gain, its response is a significant improvement over uncompensated speakers.

SHR control gain design in the presence of actuator dynamics is implemented with an empirical design guided by the above analytical model. The strategy is to find gains, $K_{P}$ and $K_{I}$, that placed the resonance at various frequencies, while maintaining the same peak amplitude. As shown above, $K_{P}$ primarily modifies SHR resonant amplitude and $K_{I}$ modifies SHR resonant frequency. Figure 3 shows simulated frequency response of the $P_{2} / D_{1}$ transfer function in the presence of actuator dynamics (Birdsong, 1999) for three sets of SHR feedback controller gains (Table 1). The controller places the resonant frequency between 110 and $150 \mathrm{~Hz}$ while maintaining a maximum peak of equal magnitude for all cases. The controller successfully achieves the goal of re-tuning the resonator with the actuator. It clearly shows that varying $K_{P}$ and $K_{I}$ results in changing the system resonant frequency and peak amplitude. 
This result indicates that the compensated acoustic actuator will perform the task of the complex boundary condition.

The SHR design has two important implementation benefits. First, the SHR is semi-active and removing the boundary condition, i.e., the controller is turned off, simply reverts the system to the nominal resonance defined by the physical dimensions of the Helmholtz resonator. When these dimensions are designed to meet nominal performance requirements, turning off the controller will only remove the variable tuning leaving the nominal tuning in tact. Second, it has the benefit that it is designed fully removed from the target system and all sensitive moving parts, microphone and actuator are located fully inside of the Helmholtz resonator not directly in the path of the fluid flow. Unlike other piping and duct noise controls, no sensors are required to be installed on the target system. This provides the advantage that debris carried by the fluid in the system will not come in direct contact with the microphone and actuator.
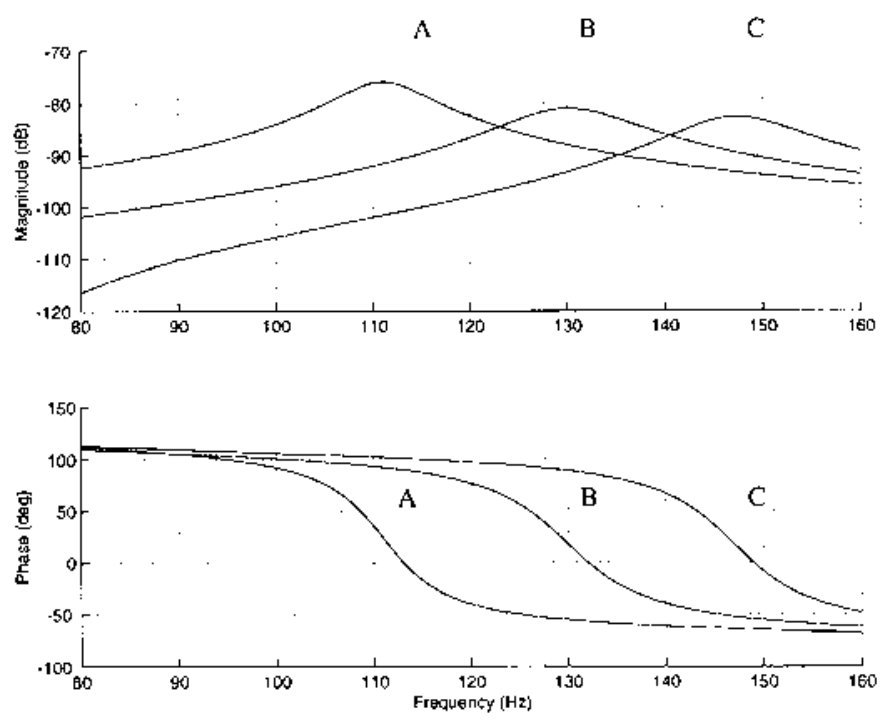

Figure 3. Simulated Closed-Loop SHR Frequency Response For /D1 With Actuator Dynamics (Birdsong, 1999)

\section{ACOUSTIC NOISE POWER FLOW - WHERE DOES THE NOISE GO?}

The system of interest is a rigid, long straight pipe or duct with constant cross sectional area, $A_{D}$, a SemiActive Helmholtz Resonator (SHR) attached to the wall, at $x=0$ and a source generating sound at one end of the duct.. Figure 4 shows a schematic of the system with arrows indicating the directions of incident, reflected, absorbed and transmitted power.

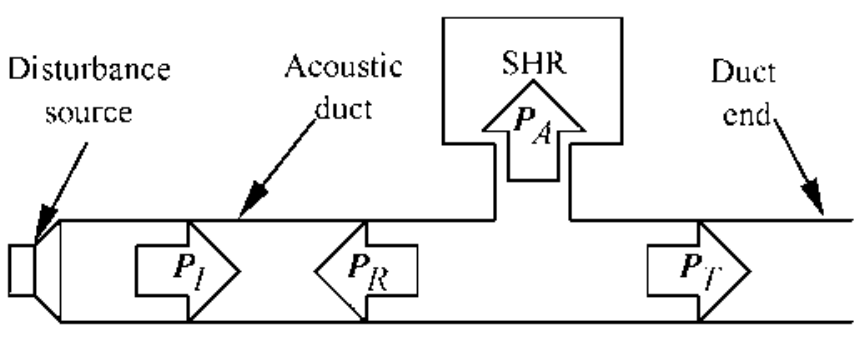

Figure 4. Schematic Diagram Of SHR And Acoustic Duct Showing Incident Power From Noise Source, Absorbed Power In SHR And Transmitted Power Towards Open End (Birdsong, 1999)

The objective of this study is to develop a relationship for the various power quantities in terms of the SHR properties. A lumped-parameter model (Pierce, 1981; Birdsong, 1999) is used which neglects dissipation in the duct, incorporates one-dimensional acoustic theory, assumes continuous pressure, and assumes that the volume velocity is conserved at the junction giving,

$$
Q_{i}=Q_{i}+Q_{i}
$$

where $Q_{I}, Q_{A}$, and $Q_{T}$ are the volume velocities $\left(\mathrm{m}^{3} / \mathrm{s}\right)$ of the incident, absorbed and transmitted paths. The pressure in the duct is continuous, so the pressure in each path at the intersection is equal,

$$
P_{1}=P_{{ }^{4}}=P_{P}
$$

where $P_{I}, P_{A}$, and $P_{T}$ are the pressures $(\mathrm{Pa})$ in the incident, absorbed, and transmitted paths. Acoustic impedance is the relationship between $P$ and $Q$ across a section. Dividing (12) by (13) gives a relationship between the acoustic impedance for each path as

$$
\chi_{I}^{-1}=\%_{\cdot 4}^{-1}+Z_{i}^{-1}
$$

where $Z_{I}, Z_{A}$, and $Z_{T}$ are the acoustic impedance $\left(\mathrm{Ns} / \mathrm{m}^{5}\right)$ of the incident, absorbed and transmitted paths respectively.

The acoustic impedance of the incident path is analyzed by considering the forward traveling and reflected pressure wave in the incident path. The total incident pressure is given by the sum of the forward and reflected wave amplitudes,

$$
P_{i}=\mathbf{P}\left(e^{-i k x}+\mathbf{R} e^{i k x}\right)
$$

where $\mathbf{P}$ is the pressure amplitude, $k$ is the wave constant $(\omega / \mathrm{c}), x$ is the spatial variable, and $\mathbf{R}$ is the pressure-amplitude reflection coefficient that gives the ratio of the reflected to incident pressure wave amplitude. The volume-velocity in the incident path is given by

$$
Q_{t}=\frac{\mathbf{P} A_{i}}{\rho c}\left(e^{-k x}-\mathbf{R} e^{i k x}\right)
$$


where $\rho$ is the density $\left(\mathrm{kg} / \mathrm{m}^{3}\right)$ of the medium, and $c$ is the speed $(\mathrm{m} / \mathrm{s})$ of sound. The quantity $\rho c / A_{D}$ can be defined as $Z_{0}$ and represents the acoustic impedance of air in a free field. The acoustic impedance in the incident path is given by the ratio of the pressure to the volumevelocity,

$$
z_{i} \equiv \frac{P_{I}}{Q_{i}}
$$

Substituting (15) and (16) into (17) gives

$$
\frac{\chi_{1}}{\%_{0}}=\frac{1+\mathbf{R}}{1-\mathbf{R}}
$$

Solving for $\mathbf{R}$ gives,

$$
\mathbf{R}=\frac{Z_{I}-Z_{11}}{Z_{I}+Z_{i !}}
$$

Substituting (14) for $Z_{I}$ in (19) gives

$$
\mathbf{R}=\frac{\left(Z_{A}^{-1}+Z_{T}^{-1}\right)^{-1}-Z_{0}}{\left(Z_{A}^{-1}+Z_{T}^{-1}\right)^{-1}+Z_{0}}
$$

These results are valid for a general values of $Z_{A}$, and $Z_{T}$, however, by considering an idealized boundary condition at the duct end at $x=L$, further analysis can be developed. Consider equal impedances in both sections of the duct such that no termination reflections occur. Under this assumption, $Z_{T}=Z_{I}=Z_{0}$ and (20) can be simplified to give the reflection coefficient, $\mathbf{R}$ in terms of $Z_{A}$ and $Z_{0}$ as,

$$
\mathbf{R}=\frac{-1}{I+\frac{2 Z_{1}}{Z_{u}}}
$$

The transmission coefficient, $\mathbf{T}$ gives the amplitude of the transmitted pressure relative to the incident pressure and is given by,

$$
\mathbf{T}=1+\mathbf{R}
$$

The fractions of reflected, transmitted and absorbed power to incident power are given by

$$
\begin{gathered}
\mathbf{P}_{\mathrm{K}} / \mathbf{P}_{\mathrm{I}}=|\mathbf{R}|^{2} \\
\mathbf{P}_{\mathrm{Y}} / \mathrm{P}_{\mathrm{I}}=|\mathbf{T}|^{2} \\
\mathbf{P}_{\mathrm{H}} / \mathbf{P}_{\mathrm{I}}=\mathrm{l}-|\mathbf{R}|^{2}-|\mathbf{T}|^{2}
\end{gathered}
$$

These equations (23), (24), and (25) are ratios of reflected, transmitted, and absorbed power relative to incident power. The ratios $\mathbf{P}_{\mathrm{R}} / \mathbf{P}_{\mathrm{I}}$ and $\mathbf{P}_{\mathrm{T}} / \mathbf{P}_{\mathrm{I}}$ can range from zero to one, and it can be shown that $P_{A} / P_{I}$ attains a maximum of one half when the ratio of $Z_{A} / Z_{T}$ is one half.

The transmission of power through the intersection depends on the impedance $Z_{\mathrm{A}}$, which can theoretically vary from 0 to $\infty$. When $Z_{A}=\infty$, the SHR acts as a rigid wall. In this case, $\mathbf{R}=0$, and $\mathbf{T}=1$, i.e., there is no reflection $\left(\mathbf{P}_{\mathrm{R}}=0\right)$ and all the power is transmitted $\left(\mathbf{P}_{\mathrm{T}}=1\right)$. This scenario is equivalent to no SHR present and the system consists of simply a rigid duct that transmits sound perfectly. When $Z_{A}=0$ the intersection behaves as an ideal "pressure release" boundary. In this case $\mathbf{R}=-1$ and $\mathbf{T}=0$, i.e., all of the incident power is reflected, $\left(\mathbf{P}_{\mathrm{R}}=1\right)$ and none is transmitted $\left(\mathbf{P}_{\mathrm{T}}=0\right)$. In reality the SHR impedance will fall between these extremes, resulting in finite values of $Z_{A}$.

Transmission Loss $(T L)$ is a measure of the power flow that is commonly used in acoustics. It is the ratio of transmitted to incident power and is given by

$$
W L=\left(\mathbf{P}_{\mathrm{r}} / \mathbf{P}_{\mathrm{L}}\right)^{-1}=\frac{\mathrm{I}}{|\mathrm{I}+\mathbf{R}|^{2}}
$$

Note that $T L$ is the inverse of $\mathbf{P}_{\mathrm{T}} / \mathbf{P}_{\mathrm{I}}$ and increases as the transmitted power is reduced relative to the incident power. For example, the pressure release condition $\left(Z_{A}=0, \mathbf{R}=-1\right)$ gives $\mathrm{TL}=\infty$.

The purpose of the SHR is to create a variable to control the power flow in the system. The SHR transfer function (11) gives the reciprocal of $Z_{A}$ in terms of acoustic parameters that are fixed, and controller gains that can be changed on line. The power flow of the duct and SHR system can be modified on line by varying $Z_{A}$ through the controller gains $K_{I}$ and $K_{P}$. For most effective behavior maximizing transmission loss $T L$, the SHR is configured to minimize

$$
Z_{.4}=\frac{P_{.1}}{Q_{2}}=\frac{P_{1}}{Q_{1}}
$$

which is the inverse of the SHR transfer function (11). This objective is accomplished by maximizing the resonant amplitude of the SHR at the frequency of incident tonal noise. A set of gains which adjusts the SHR resonant frequency to the incident noise frequency and then maximizes that resonant amplitude will generate a maximum transmission loss past the SHR.

\section{EXPERIMENTAL VALIDATION}

An experimental apparatus was constructed to validate the theoretical model and to demonstrate the noise reduction capability of the Semi-active Helmholtz Resonator (SHR) device. The experimental SHR setup consisted of two components: a Helmholtz resonator cavity and a microphone-compensated actuator system. Figure 5 shows a photograph of the SHR connected to an acoustic duct and Figure 6 shows a schematic of the setup. A cylindrical Helmholtz resonator cavity was 
constructed from PVC with dimensions $0.075 \mathrm{~m}$ in diameter and $0.15 \mathrm{~m}$ in length. A cylindrical neck with dimensions $0.018 \mathrm{~m}$ diameter and $0.01 \mathrm{~m}$ in length, was fitted on one face of the cavity. The microphonecompensated actuator system consisted of a half inch B\&K type 4155 microphone sealed through the wall of the cavity. A D-Space Model \#1102 floating point, digital signal processor (DSP) was used to implement the speaker compensation and the controller, and an acoustic actuator was sealed in the opposite face of the cavity. A DSP sampling rate of $5 \mathrm{kHz}$ was used for all experiments.

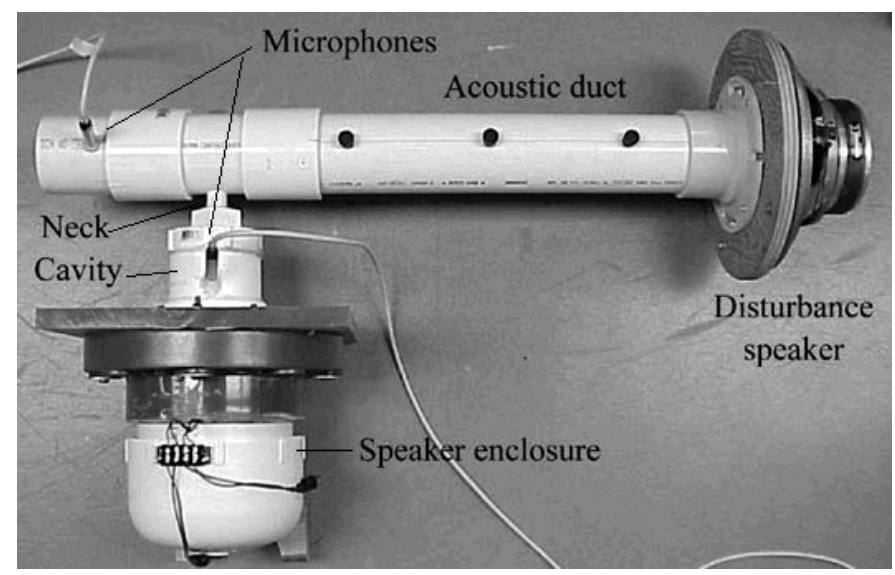

Figure 5. Photograph Of SHR Connected To An Acoustic Duct With A Second Audio Speaker To Inject Noise (Birdsong, 1999)

In all phases of the system design, the device was separated from any primary acoustic system as is customary in resonator design. In the absence of a disturbance pressure, , the system was disturbed electrically by the signal D1 injected via the actuator input voltage.

The actuator was compensated to improve the speaker performance, but finite gain and phase errors in the actuator response effected the system. A speaker velocity estimator (Birdsong and Radcliffe, 1999; Radcliffe and Gogate, 1996) was implemented by combining the voltage in the secondary coil with the current in the primary coil. After the speaker compensator was implemented, the closed-loop speaker compensation was considered a single block in the SHR, and all subsequent open and closed-loop SHR experiments included closed-loop speaker compensation.

The PI controller was implemented and the closed-loop SHR response was recorded with controller gains $K_{P}=K_{I}=0$. In this configuration, the compensator attempts to hold the speaker face fixed in the presence of the disturbance. White noise was input as the disturbance to the system and the transfer function of $P_{2} / D_{1}$ was measured using a Hewlett Packard dynamic signal analyzer model \#35660A. The results indicated that a resonant peak occurred at $120 \mathrm{~Hz}$, however there was significant damping in the system which reduced the peak amplitude. This damping was attributed to mechanical damping in the form of friction and electrical power dissipation in the current sensing resistor, $R_{m}$, in the speaker compensator.

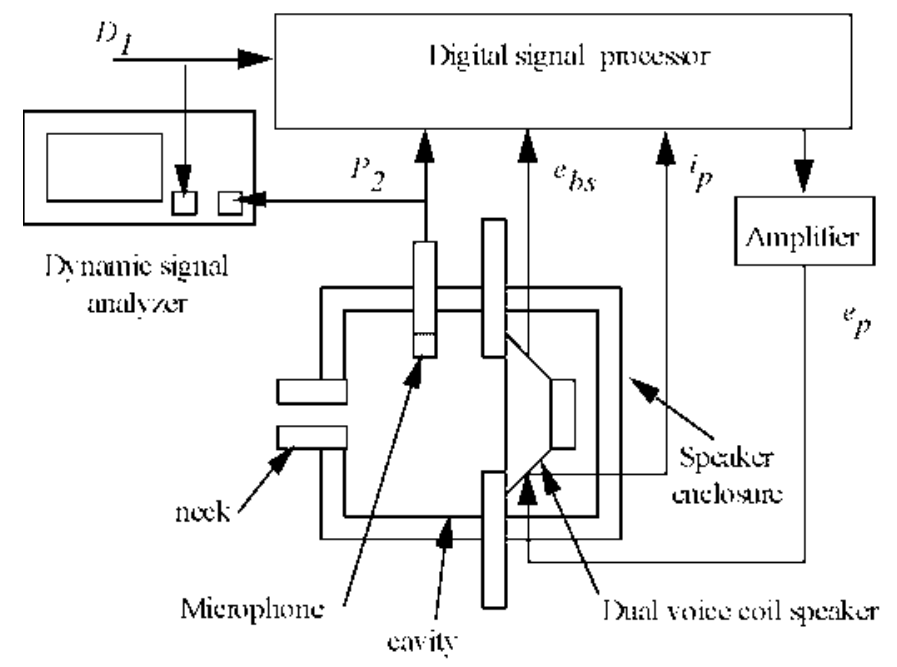

Figure 6. Schematic Diagram Of Experimental SHR Apparatus (Birdsong, 1999)

The closed-loop response was then measured with various non-zero controller gains. As predicted by the model, the analytical mapping from $K_{P}$ and $K_{I}$ to the resonant frequency and peak amplitude, (9) - (12), did not produce the desired results. This was attributed to the deviation of the actuator from the ideal model. Even with the compensator, the effects of the speaker dynamics were not sufficiently minimized.

The empirical technique (Birdsong, 1999) was used in place of the analytical mapping to tune the system in the presence of significant actuator dynamics. The PI controller design was based on qualitative information learned from the model. The objective was to find gains, $K_{P}$ and $K_{I}$, that placed the resonance at various frequencies, while maintaining the same level of damping. The data was collected by fixing $K_{I}$, searching for a $K_{P}$ that produced the desired peak amplitude, and recording the resonant frequency. The gains $K_{P}$ and $K_{I}$ are plotted against resonant frequency in Figure 7 . Note that although there is a difference in the magnitude, the overall trends of these graphs agree with the data derived from the model in Table 2 . The $K_{P}$ gain is negative for all values of $\omega_{c}$ with the most negative value at the nominal resonant frequency $(130 \mathrm{~Hz})$. Only a $10 \%$ change in $K_{P}$ is required for the entire range of $\omega_{n}$. The model predicted that $K_{P}$ had no change in the range of $\omega_{n}=110$ to $145 \mathrm{~Hz}$. The $K_{I}$ gain ranges from -100 to 200 and passes through zero at the nominal resonant frequency. 

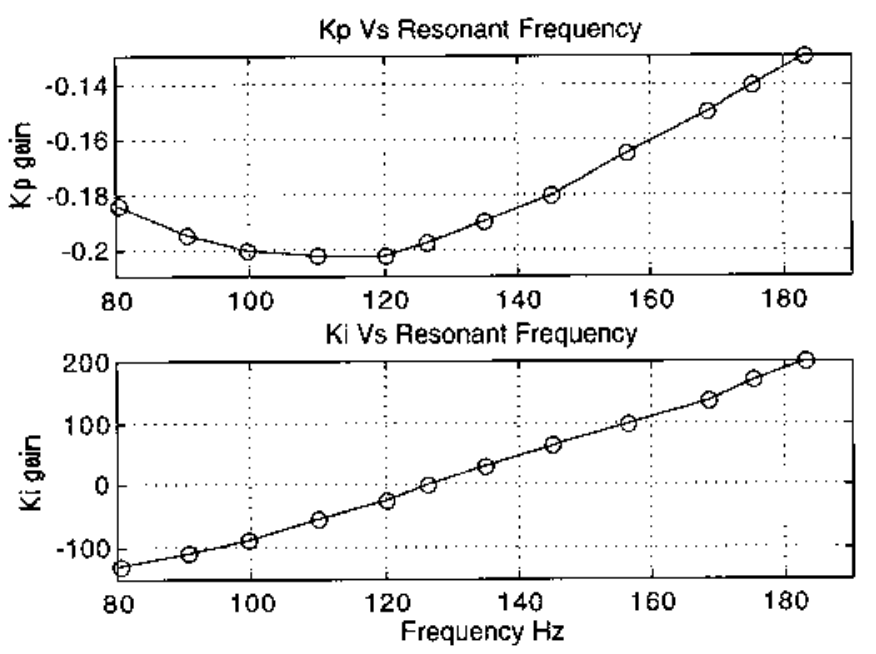

Figure 7. Graph of $K_{P}$ and $\mathrm{K}_{\mathrm{I}}$ vs. $\omega_{n}$ Measured Experimentally (Birdsong, 1999)

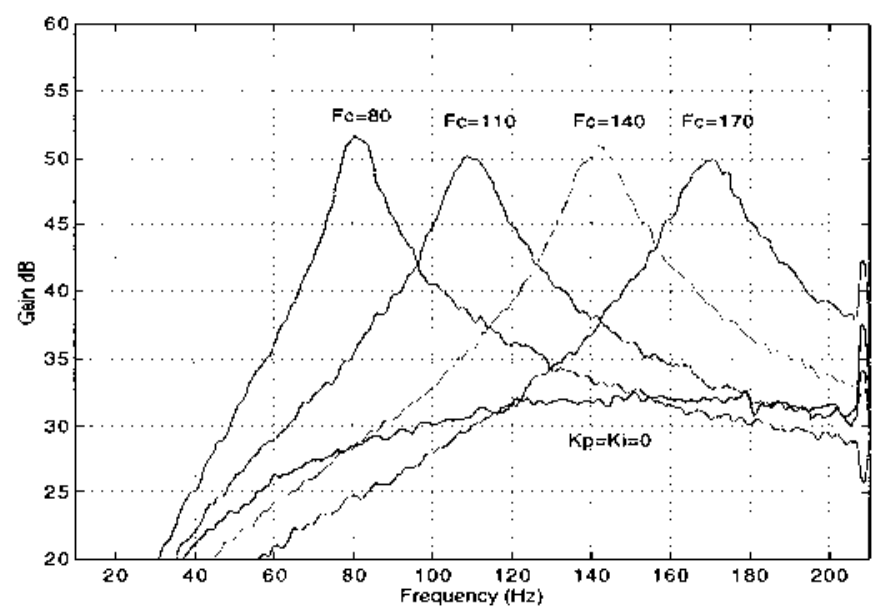

Figure 8. Experimental Measurements of SHR frequency response $P_{2} / D$. The tests show the SHR configured with $\omega_{n}=80,110,140,170 \mathrm{~Hz}$ and gains $K_{P}=$ $K_{I}=0$ (Birdsong, 1999)

The tuning capabilities of the device are illustrated by the closed-loop frequency response measurements (Figure 8). The five graphs show the resonant peak for five separate experiments with $\omega_{\mathrm{n}}=80,110,140$, and $170 \mathrm{~Hz}$ and one graph for the controller gains set to zero. Note that the SHR with the gains set to zero is over-damped with approximately $45 \%$ damping. With the controller turned on, a resonance is exhibited and positioned at the desired frequency with approximately $5 \%$ damping for all cases. The high resonant amplitude associated with this low damping value maximizes the effectiveness of the SHR as discussed previously.

\section{CONCLUSIONS}

This work presented an electronically tuned semi-active Helmholtz resonator that can be used to quiet noise in enclosed acoustic systems. It represents a powerful new tool in tuning acoustic systems. It adds a nominal resonance to an acoustic system and allows the resonance frequency and peak magnitude to be changed on-line continuously over a range of frequencies. It has advantages over other technologies in that it does not add significant mechanical complexity, it is fault tolerant, and the design places the sensitive sensor and actuator away from the direct path of the process.

\section{ACKNOWLEDGEMENTS}

This work was funded by Visteon Corporation and with the technical assistance of Dr. Lakhi Goenka at Visteon. The work was conducted as part of the Ph.D. dissertation of Dr. Charles Birdsong.

\section{REFERENCES}

1. Bedout, Francheck, et. Al. 1997, "Adaptive-Passive Noise Control with Self-Tuning Helmholtz Resonators," Journal of Sound and Vibration, v 202, p109-123.

2. Birdsong, C. B., and Radcliffe, C. J., 1999, "A Compensated Acoustic Actuator for Systems with Strong Dynamic Pressure Coupling," Journal of Vibrations and Acoustics, Vol. 121, pp. 89-94.

3. Birdsong C. B., 1999, A Semi-Active Helmholtz Resonator, Ph.D. dissertation, Michigan State University, East Lansing, Michigan.

4. Birdsong, C., 1996, A Compensated Actuator for an Acoustic Duct, Masters Thesis, Michigan State University.

5. Heidelberg, L. J., and Gordon, E. B., 1989, "Acoustic Evaluation of the Helmholtz Resonator Treatment in the NASA Lewis 8- by 6-Foot Supersonic Wind Tunnel," NASA technical memorandum ; 101407.

6. Hsomi, M., Goawao, S., Imagawa, T., and Hokazono, Y., 1993, "Development of Exhaust Manifold Muffler," SAE Special Publications New Engine Design and Engine Component Technology International Congress and Exposition Mar 1-5, 1972, Detroit

7. Pierce, Allan D., 1981, Acoustics : An Introduction To Its Physical Principles And Applications, New York : McGraw-Hill Book Co.

8. Radcliffe C. J., and Gogate, S. D., 1996, "Velocity Feedback Compensation of Electromechanical Speakers for Acoustic Applications," International Federation of Automatic Control, Triennial World Congress, July.

9. Radcliffe C.J., and Gogate S.D., Hall G., 1994, "Development of an Active Acoustic Sink (AAS) for Noise Control Applications," Active Control of Vibrations and Noise, ASME.IMECE, Nov.

10. Temkin, 1981, Elements of Acoustics, John Wiley \& Sons, Inc. 